\title{
Midlife improvements in financial situation are associated with a reduced dementia risk later in life: the CAIDE 30-year study
}

\author{
S. Sindi, ${ }^{1,2}$ (1) A. Darin-Mattsson, ${ }^{3}$ I. Kåreholt, $1,3,4$ J. Kulmala, ${ }^{1,5}$ T. Ngandu, ${ }^{5}$ \\ T. Laatikainen, ${ }^{5,6,7}$ H. Soininen, ${ }^{8,9}$ and M. Kivipelto $0^{1,2,9,10,11}$ \\ ${ }^{1}$ Division of Clinical Geriatrics, Center for Alzheimer Research, Karolinska Institutet and Karolinska University Hospital, Stockholm, Sweden \\ ${ }^{2}$ Neuroepidemiology and Ageing Research Unit, School of Public Health, Imperial College London, London, United Kingdom \\ ${ }^{3}$ Aging Research Center, Karolinska Institutet and Stockholm University, Stockholm, Sweden \\ ${ }^{4}$ Institute of Gerontology, School of Health and Welfare, Aging Research Network - fönköping (ARN-f), fönköping University, fönköping, Sweden \\ ${ }^{5}$ Chronic Disease Prevention Unit, National Institute for Health and Welfare, Helsinki, Finland \\ ${ }^{6}$ Institute of Public Health and Clinical Nutrition, University of Eastern Finland, Kuopio, Finland \\ ${ }^{7}$ Hospital District of North Karelia, Foensuu, Finland \\ ${ }^{8}$ Neurocenter, Neurology, Kuopio University Hospital, Kuopio, Finland \\ ${ }^{9}$ Institute of Clinical Medicine, Neurology, University of Eastern Finland, Kuopio, Finland \\ ${ }^{10}$ Theme Aging, Karolinska University Hospital, Stockholm, Sweden \\ ${ }^{11}$ Stockholms Sjukhem, Research and Development Unit, Stockholm, Sweden
}

\section{ABSTRACT}

Objectives: Perceived financial strain is associated with various health conditions, but it is unknown whether it is associated with an increased risk for dementia. The goal is to examine the associations between midlife perceptions of financial situation and dementia risk later in life.

Methods: Participants were derived from the Cardiovascular Risk Factors, Aging, and Dementia populationbased cohort study $(n=2000)$ (between 1972 and 1987, baseline mean age 50 years) in Finland. Participants returned for two re-examinations in late life (in 1998 and 2005-2008, mean age 71 and 78 years). In this study, 1442 subjects that participated in at least one re-examination (mean total follow-up 25 years) were included in analyses. Financial strain was measured using two questions in midlife on perceptions of financial situation and perceptions of changes in financial situation. For each question, participants were categorized into three groups reporting improvement, worsening, or stability, with the latter set as the reference group. Analyses were adjusted for potential confounding factors.

Results: The group reporting better financial situation had a reduced risk for dementia (fully adjusted model: odds ratio (OR): $0.53,95 \%$ confidence interval (CI): 0.33-0.86). In contrast, the group reporting worse financial situation did not have an increased risk for dementia (OR: 1.04, 95\% CI: 0.53-2.02). Analyses on perceptions of current financial situation showed that the groups reporting satisfaction or dissatisfaction with financial situation did not differ in risk for dementia.

Conclusion: This study is the first to show that midlife improvements in financial situation are associated with a reduced dementia risk later in life. Potential pathways related to stress reduction, improved lifestyle, and potential biological mechanisms are discussed.

Key words: financial strain, stress, cognitive impairment, midlife risk factors, midlife protective factors

\section{Introduction}

Lower income or socioeconomic position in late life is associated with an increased risk for dementia

Correspondence should be addressed to: Shireen Sindi, Department of Neurobiology, Care Sciences and Society (NVS), Karolinska Institutet, Division of Clinical Geriatrics, Center for Alzheimer Research, Aging Research Center, Karolinska Universitetssjukhuset, Karolinska Vägen 37 A, QA32, 17164 Solna, Sweden. Phone: + 46 735508703. Email: shireen.sindi@ki.se. Received 28 Dec 2018; revision requested 28 Jan 2019; revised version received 09 Oct 2019; accepted 11 Oct 2019. First published online 25 November 2019.
(Anttila et al., 2002; Bootsma-van der Wiel et al., 2005; Evans et al., 1997; Fischer et al., 2009; Sattler et al., 2012). A few studies have investigated the associations between midlife income and dementia risk later in life, showing that midlife income was not associated with later dementia or dementia mortality risk (Anttila et al., 2002; Strand et al., 2015). While the aforementioned studies have focused on objective measures of income, little attention has been given to (potentially stressful) perceptions of financial situation and dementia risk. Studies on 
stress provide evidence for interindividual variability in perceptions and reactivity to the same stressors (Ebner and Singewald, 2017; Folkman, 2013; Southwick et al., 2016). This implies that individuals with the same income may experience their financial situation differently. It is therefore important to investigate not only whether income (objective measure) alters dementia risk, but also whether the perceptions of one's financial situation (subjective measure) play a role.

Perceived financial strain increases distress and preoccupations (e.g. regarding not being able to pay for food/housing bills or health-related needs/ activities) (Mirowsky and Ross, 2003), or it may lead to other stressful experiences such as interpersonal/family conflicts (Palta et al., 2015; Pearlin et al., 1997). Various sources of stress have gained attention for their important role as dementia risk factors (Hakansson et al., 2015; Johansson et al., 2010; Johansson et al., 2013; Sindi et al., 2016). To date, no studies have assessed whether perceived financial strain is associated with dementia risk.

The aim of this study was to examine the associations between midlife perceptions of financial situation and perceptions of changes in financial situation, both improvement and worsening, and dementia risk later in life assessed during two re-examinations, in a population-based cohort study (average total follow-up 28.5 years).

\section{Materials and methods}

\section{Sample}

The Cardiovascular Risk Factors, Aging and Dementia (CAIDE) study participants were first examined at midlife (baseline) in the North Karelia Project and the FINMONICA (later FINRISK) population-based studies in Finland. Individuals underwent baseline assessments in one of the following years: 1972, 1977, 1982, or 1987, and over the years the sample size ranged from 7927 to 13,699 (Borodulin et al., 2017; Puska, 2010). Baseline participation rates ranged between $82 \%$ and $90 \%$. The CAIDE study targeted older adults aged 65-79 years at the end of 1997, living in the cities of Kuopio and Joensuu. Of 3559 eligible individuals, $2684(75.4 \%)$ remained (with 875 (24.6\%) having died before the end of 1997).

In 1998, a random sample of 2000 survivors aged 65-79 years were invited for the first re-examination. In total, 1449 individuals $(72.5 \%)$ participated and 1409 performed the cognitive assessments. The mean follow-up time was 21 years $(\mathrm{SD}=4.9)$. Participants returned for a second re-examination between 2005 and 2008. In 2005, 1426 (of the original sample of 2000) were alive and living in the same region. When invited, $909(63.7 \%)$ of these accepted to participate and 852 completed the cognitive assessments. A total of 1511 individuals participated in at least one re-examination, and 750 participated in both. Mean ages at the different time points are displayed in Table 1. Local ethics committees in Kuopio and Joensuu approved the CAIDE study, which complies with the Declaration of Helsinki. Informed consent was obtained from all individual participants.

\section{Measurement of financial situation}

Financial situation in midlife (baseline) was measured using the following two questions with 5-point likert scales. Each question was analyzed separately:

(1) Satisfaction with financial situation at midlife: The question asked: 'How satisfied are you with your financial situation?'. Response options were: 1 - Very satisfied, 2 - Satisfied, 3 - Somewhat satisfied, 4 - Dissatisfied, 5 - Very dissatisfied.

This variable was recategorized into the following three categories: (1) Satisfied (combining choices 1 and 2), (2) Somewhat satisfied (choice 3), (3) Dissatisfied (combining choices 4 and 5).

(2) Change in financial situation at midlife: The question asked: 'Is your financial situation now better or worse than before?'. Response options were: 1 - Much better, 2 - A little bit better, 3 - Same as before, 4 - A little bit worse, 5 - Much worse.

This variable was recategorized into the following three categories: (1) Better (combining choices 1 and 2), (2) Same as before (choice 3), (3) Worse (combining choices 4 and 5).

The Pearson product-moment correlation coefficient was calculated to assess the association between the two questions on financial situation. There was a significant positive association between the two questions, $r=0.381, p=<0.001$.

\section{Diagnosis of dementia and Alzheimer's disease in the CAIDE study}

Cognitive performance was assessed in both re-examinations using a three-step protocol for dementia diagnosis: screening phase, clinical phase, and differential diagnostic phase. At the first reexamination (1998), participants who scored $\leq 24$ on the Mini-Mental State Examination (MMSE) (Folstein et al., 1975) at screening were referred to the clinical phase for further examinations. At the second re-examination (2005-2008), participants were referred to the clinical phase if they scored 
Table 1. Sociodemographic and clinical characteristics of participants included in the analyses at the first and second re-examinations

\begin{tabular}{|c|c|c|c|c|}
\hline \multirow[b]{2}{*}{ CHARACTERISTICS } & \multicolumn{2}{|c|}{$\begin{array}{l}\text { FIRST RE-EXAMINATION } \\
\text { (1998) }\end{array}$} & \multicolumn{2}{|c|}{$\begin{array}{l}\text { SECOND RE-EXAMINATION } \\
(2005-2008)\end{array}$} \\
\hline & $n$ & MEAN (SD) OR $n(\%)$ & $n$ & MEAN (SD) OR $n(\%)$ \\
\hline Dementia cases & 1399 & $60(4.3 \%)$ & 731 & $44(6.0 \%)$ \\
\hline Baseline age & 1399 & $50.3(6.0)$ & 731 & $49.4(6.0)$ \\
\hline Age at follow-up & 1399 & $71.3(4.0)$ & 731 & $78.4(3.7)$ \\
\hline Follow-up time & 1398 & $20.9(4.9)$ & 729 & $29.0(5.0)$ \\
\hline Sex & 1399 & & & \\
\hline Women & & $869(62.1 \%)$ & 731 & $476(65.1 \%)$ \\
\hline Education (years) & 1378 & $8.6(3.4)$ & 720 & $9.2(3.5)$ \\
\hline$A P O E \varepsilon 4$ allele & 1364 & & & \\
\hline Carrier & & $483(35.4 \%)$ & 718 & $228(31.8 \%)$ \\
\hline $\begin{array}{l}\text { Change in economical situation at } \\
\text { midlife (range } 1-5)^{\mathrm{a}}\end{array}$ & 1399 & $2.5(0.9)$ & 731 & $2.5(0.9)$ \\
\hline $\begin{array}{l}\text { Satisfaction with economical situation } \\
\text { at midlife (range } 1-5)^{\mathrm{b}}\end{array}$ & 1396 & $2.7(0.7)$ & 728 & $2.7(0.8)$ \\
\hline Type of occupation & 1347 & & 705 & \\
\hline White collar & & $643(47.7 \%)$ & & $374(53.1 \%)$ \\
\hline Blue collar & & $396(29.4 \%)$ & & $170(24.1 \%)$ \\
\hline Housewives & & $205(15.2 \%)$ & & $109(15.5 \%)$ \\
\hline Other & & $103(7.7 \%)$ & & $52(7.4 \%)$ \\
\hline $\begin{array}{l}\text { Midlife cardio/cerebrovascular/ } \\
\text { respiratory conditions }\end{array}$ & 1399 & & & \\
\hline Yes & & $68(4.9 \%)$ & 731 & $26(3.7 \%)$ \\
\hline Midlife hopelessness & 1361 & & 712 & \\
\hline Yes & & $578(42.5 \%)$ & & $273(38.3 \%)$ \\
\hline
\end{tabular}

$\leq 24$ points on MMSE, or had a decrease $\geq 3$ points on MMSE since 1998, or if they scored $<70 \%$ in delayed recall on the Consortium to Establish a Registry for Alzheimer's Disease word list (Morris et al., 1989), or if the participant's informant expressed concerns regarding his/her cognition. These criteria were added to increase sensitivity for the detection of milder cognitive impairment. The clinical phase (at both re-examinations) included detailed neurological, neuropsychological, and cardiovascular examinations, and the differential diagnostic phase included brain imaging (magnetic resonance imaging/computerized tomography), blood tests, and if needed cerebrospinal fluid analysis, and electrocardiogram. A review board (including the physician, neuropsychologist, and senior neurologist) used all assessment information and ascertained the primary diagnosis. Dementia diagnosis was based on the Diagnostic and Statistical Manual of Mental Disorders criteria (1994). For Alzheimer's disease diagnoses, the criteria used were based on the National Institute of Neurological and Communicative Disorders and Stroke and the Alzheimer's Disease and Related Disorders Association (NINCDS-ADRDA) (McKhann et al., 1984).

\section{Other assessments}

Standardized assessments and survey methods at baseline (midlife) adhere to international guidelines and the World Health OrganizationMultinational MONItoring of trends and determinants in cArdiovascular disease (MONICA) protocol (Pajak et al., 1988). Re-examination surveys were similar and comparable with baseline surveys. The surveys involved questionnaires on sociodemographic factors, health status, medical history, health-related behaviors, and psychological factors. In the analyses, we included the following covariates measured at baseline: age, sex, education, apolipoprotein E (APOE) $\varepsilon 4$ allele status, cardio/cerebrovascular and respiratory conditions, type of occupation, and hopelessness.

Main lifetime occupation was measured by asking individuals to select their longest-held occupation from these categories: office/service, farming/forestry, mining/industrial/construction work, housewives, or other. Hopelessness was measured using two questions described recently (Hakansson et al., 2015), using a 5-point Likert scale: "I feel that it is impossible to reach the goals I would like to strive for" and "The future seems to me to be hopeless, and I cannot believe that things 
are changing for the better". For measures of income at baseline, participants selected their total household income before taxes from nine categories. The categories changed somewhat between the data collections due to changes in income level. We categorized these nine categories as low, medium, and high income. The three categories had approximately the same distribution each survey time. A venous blood sample was taken for measures of biomarkers, including APOE genotype from blood leukocytes analyzed using $\mathrm{hHaI}$ digestion and polymerase chain reaction (Tsukamoto et al., 1993). The Hospital Discharge Register was used to obtain information on diagnoses of respiratory and cardio/cerebrovascular conditions (chronic obstructive pulmonary disease, asthma, coronary artery disease, stroke, myocardial infarction, atrial fibrillation, cardiovascular surgery, heart failure, or diabetes).

\section{Statistical analyses}

Analyses were performed using Stata 13.0 (Stata Corp, College Station, TX, U.S.A). The significance level for all analyses was set at $p<0.05$. We carried out separate analyses for the associations between the midlife satisfaction with financial situation and midlife change in financial situation and late-life dementia, using dementia diagnoses obtained in the CAIDE examinations $(n=1500)$.

In order to maximize the sample size, all subjects with a dementia diagnosis in at least one re-examination were included in the analyses. The analytical sample was reduced to 1442 due to nonresponse to the questions on financial situation. Data were organized in what is often referred to as long format. Persons with dementia diagnoses both at first and second re-examination, and dementiafree at the first follow-up, are included twice in the data set with one observation for the first re-examination and one observation for the second re-examination. A few people had dementia at the first re-examination and were still alive at the second re-examination. They were excluded from the second re-examination. This means that each person can only be included once with dementia. We carried out logistic regressions for the associations between midlife financial strain and dementia in late life (at the first or second re-examinations), and reported results as odds ratios (ORs) and $95 \%$ confidence intervals (CIs). Using data in long format, but with persons with dementia included only once, corresponds to a hazard regression with (two) discrete time points. With such analyses one obtains the same results with the long format as with the short format, and time is coded at specific discrete values, for example, 1 and 2 . The difference is that the current results are presented as ORs and the results from hazard regressions are presented as relative risks. These results correspond to the analysis of the pooled risk of having incident dementia (since previous time point) at first and second re-examination (Allison, 1982; Singer and Willett, 1993). All analyses were adjusted for basic confounders: age, sex, education (total years), annual baseline household income, baseline cohort, ApoE $\varepsilon 4$ allele (all included in Models 1, 2, and 3). Subsequent analysis (Models 2) was additionally adjusted for cardio/cerebrovascular conditions and hopelessness. Additional analysis (Models 3) additionally adjusted for type of occupation (categorized as white-collar occupations (office/ service), blue-collar occupations (farming/forestry, mining/industrial/construction work), housewives, or other occupations.

\section{Results}

\section{Sample characteristics}

Table 1 presents the sociodemographic and clinical characteristics of the participants included in the analyses in both re-examinations. Data for categorical variables are reported as proportions, while data for continuous variables are reported as means (SD).

MidlifE FinANCiAL SITUATION AND RISK OF DEMENTIA

Satisfaction with Financial Situation and Dementia. In response to the question 'How satisfied are you with your financial situation?', the reference group was set as the group that responded with 'somewhat satisfied'. Analyses showed that the group reporting satisfaction with financial situation (very satisfied or satisfied) or the group reporting dissatisfaction with financial situation (very dissatisfied or dissatisfied) did not differ in risk for dementia (Table 2).

Change in Financial Situation and Dementia. In response to the question 'Is your financial situation now better or worse than before?', the reference group was set as the one who responded with 'same as before'. Table 3 shows the descriptive characteristics of the three groups reporting a better, similar, or worse financial situation than before.

Associations between change in financial situation at midlife and dementia are shown in Table 2. 
Table 2. The associations between financial situation and late-life dementia

\begin{tabular}{|c|c|c|c|}
\hline & MODEL 1 & MODEL 2 & MODEL 3 \\
\hline & OR $(95 \% \mathrm{CI})$ & OR $(95 \% \mathrm{CI})$ & OR $(95 \% \mathrm{CI})$ \\
\hline \multicolumn{4}{|c|}{ Satisfaction with financial situation at midlife } \\
\hline Satisfied with financial situation & $0.94(0.58-1.53)$ & $0.99(0.60-1.64)$ & $1.04(0.62-1.75)$ \\
\hline Somewhat satisfied (ref) & 1.0 & 1.0 & 1.0 \\
\hline Dissatisfied with financial situation & $1.10(0.51-2.37)$ & $1.02(0.47-2.21)$ & $0.90(0.38-2.13)$ \\
\hline \multicolumn{4}{|l|}{ Change in financial situation at midlife } \\
\hline Better financial situation & $0.53(0.33-0.86)$ & $0.56(0.35-0.92)$ & $0.53(0.32-0.87)$ \\
\hline Same as before (ref) & 1.0 & 1.0 & 1.0 \\
\hline Worse financial situation & $1.03(0.53-1.99)$ & $1.04(0.53-2.02)$ & $1.04(0.53-2.02)$ \\
\hline
\end{tabular}

Results with $\mathrm{p}<0.05$ are presented in bold.

Results from logistic regressions presented as odds ratios (OR).

Model 1: age, sex, education, midlife income, cohort, apoe4.

Model 2: Model $1+$ cardio/cerebrovascular/respiratory conditions, hopelessness.

Model 3: Model $2+$ occupation type.

Table 3. Sociodemographic and clinical characteristics of participants included in the analyses at the first and second re-examinations

\begin{tabular}{|c|c|c|c|c|c|c|c|}
\hline \multirow[b]{3}{*}{ CHARACTERISTICS } & \multicolumn{6}{|c|}{ CHANGE IN FINANCIAL SITUATION } & \multirow[b]{3}{*}{$p$} \\
\hline & \multicolumn{2}{|c|}{$\begin{array}{l}\text { BETTER THAN } \\
\text { BEFORE }\end{array}$} & \multicolumn{2}{|c|}{ SAME AS BEFORE } & \multicolumn{2}{|c|}{ WORSE THAN BEFORE } & \\
\hline & $n$ & $\begin{array}{c}\text { MEAN (SD) OR } \\
n(\%)\end{array}$ & $n$ & $\begin{array}{c}\text { MEAN (SD) OR } \\
n(\%)\end{array}$ & $n$ & $\begin{array}{c}\text { MEAN (SD) OR } \\
n(\%)\end{array}$ & \\
\hline Dementia & 693 & $37(5.3)$ & 580 & $50(8.6)$ & 169 & $17(10.1)$ & 0.025 \\
\hline Baseline age & 693 & $50.2(6.0)$ & 580 & $50.6(6.2)$ & 169 & $50.4(5.8)$ & NS \\
\hline Age at follow-up & 693 & $71.2(4.0)$ & 580 & $71.2(3.8)$ & 169 & $71.8(4.2)$ & NS \\
\hline Follow-up time & 693 & $21.0(4.7)$ & 580 & $20.6(5.1)$ & 169 & $21.4(4.8)$ & NS \\
\hline Sex & 1399 & & & & & & \\
\hline Women & - & $420(61.8 \%)$ & - & $341(61.2 \%)$ & - & $108(66.7 \%)$ & NS \\
\hline Education (years) & 686 & $8.4(3.3)$ & 569 & $8.8(3.7)$ & 167 & $8.9(3.4)$ & NS \\
\hline$A P O E \varepsilon 4$ allele & 1364 & & & & & & \\
\hline Carrier & - & $244(36.6 \%)$ & - & $194(35.6 \%)$ & - & $45(29.6 \%)$ & NS \\
\hline Type of occupation & 1347 & & & & & & NS \\
\hline White collar & - & $324(48.9 \%)$ & - & $259(48.5 \%)$ & - & $60(39.7 \%)$ & \\
\hline Blue collar & - & $181(27.3 \%)$ & - & $167(31.3 \%)$ & - & $48(31.8 \%)$ & \\
\hline Housewives & - & $107(16.2 \%)$ & - & $70(13.1 \%)$ & - & $28(18.5 \%)$ & \\
\hline Other & - & $50(7.6 \%)$ & - & $38(7.1 \%)$ & - & $15(9.9 \%)$ & \\
\hline $\begin{array}{l}\text { Midlife cardio/cerebrovascular/ } \\
\text { respiratory conditions }\end{array}$ & 1399 & & & & & & \\
\hline Yes & - & $31(4.6 \%)$ & - & $28(5.0 \%)$ & - & $9(5.6 \%)$ & NS \\
\hline Midlife hopelessness & 1361 & & & & & & \\
\hline Yes & - & $249(37.7 \%)$ & - & $239(44.1 \%)$ & - & $90(56.6 \%)$ & $<0.001$ \\
\hline
\end{tabular}

Analyses showed that the group reporting an improvement in financial situation (much better or a little better) had a reduced risk for dementia in all models (fully adjusted Model 3: OR: 0.53, 95\% CI: 0.33-0.86). In contrast, the group reporting worse financial situation (much worse or little bit worse) did not have an increased dementia risk (fully adjusted Model 3: OR: 1.03, 95\% CI: 0.53-2.0).

\section{Discussion}

This study examined the associations between midlife perceptions of financial situation and perception of changes in financial situation and dementia risk later in life. The results showed that individuals who reported better financial situation had a lower risk for dementia, when compared 
with individuals who reported no change in financial situation, or individuals who reported worse financial situation. These associations remained robust after adjusting for several potential confounding factors, including education, income, and occupation type. In contrast, the level of satisfaction with one's current financial situation was not associated with later dementia risk. To the best of our knowledge, this study is the first to report such associations between self-reported improved financial situation and dementia risk.

It was interesting to find that the associations between self-reported improved financial situation and dementia were present despite adjusting for income, which may suggest that the association is independent of actual income. This is consistent with previous findings showing that while perceived financial strain or perceived income adequacy is related to income, they may also be independent (Kahn and Pearlin, 2006). It also supports earlier findings showing that objective measures are insufficient proxies of socioeconomic status and financial strain (Matthews et al., 2005; Sun et al., 2009). For example, individuals with the same income may experience different levels of financial strain due to differences in budgeting skills, the proportion of capital available for daily use (e.g. if it is invested in properties/shares) (Matthews et al., 2005), expectations, or customs. This study adds to the literature on midlife income and dementia risk. While objective measures of income are not associated with dementia risk or dementia-related mortality (Anttila et al., 2002; Strand et al., 2015), the results from the present study suggest that perceived financial situation is associated with dementia risk. Subjective perceptions of financial situation may play a role in shaping dementia risk, in addition to more objective indicators of income.

A positive perception of one's financial situation may be associated with a lower dementia risk through several pathways. One pathway is through reducing stress levels, which have previously been shown to be associated with an elevated dementia risk (Johansson et al., 2010; Sindi et al., 2016). Positive perceptions of one's financial situation may, for example, lead to perceptions of higher control and predictability of one's financial situation, which reduce the experienced stress levels. Another potential pathway is through the impact of perceived financial situation on lifestyle. Health behaviors such as physical inactivity may partly explain socioeconomic inequalities in health and old age (Pampel et al., 2010; Shaw et al., 2014). Positive perceptions of one's financial situation may promote healthier nutrition and physical/social activities, which are modifiable lifestyle factors associated with a reduced risk for dementia (Sindi et al., 2015). In terms of clinical implications, individuals who perceive their finances more negatively may be encouraged to engage in strategies that increase their locus of control and increase their perceptions of control and predictability regarding their finances. They may also benefit from advancing specific skills such as budgeting, financial management, and long-term planning, which further increase their sense of control and may promote a positive perception toward their finances. It would also be interesting for future research to investigate how personality traits (e.g. optimism) or anxiety traits modify these associations.

Several physiological mechanisms have been reported to be associated with perceived financial strain. Studies have shown that financial strain is associated with stress hormone (cortisol) levels. The cortisol awakening response was shown to be lower among men who reported an improvement in financial strain, compared with men who reported a worsening or no change (Steptoe et al., 2005). It was also shown that women reporting high financial strain had higher total cortisol levels, including high evening levels, than women reporting low financial strain. Neuroimaging findings showed that compared with individuals who reported no financial strain, those who reported financial strain had smaller hippocampal and amygdalar volumes; the key brain regions involved in cognition and emotional regulation (Butterworth et al., 2012). Financial strain has also been associated with increased proinflammatory markers (e.g. C-reactive proteins [CRP], interleukin-6 [IL-6]), and oxidative stress (Palta et al., 2015; Steffen et al., 2016). Various indicators of socioeconomic inequalities have also been associated with allostatic load, an index incorporating biomarkers from multiple systems to obtain a measure of chronic stress and subsequent 'wear and tear' on the body and brain (Juster 2016). While most of these aforementioned studies do not specifically address an improvement in perceived financial situation, it may be speculated that it is associated with a reduction in these risk factors. In the current study, it is unclear why worse financial situation was not associated with an increased risk for dementia. It may be speculated that the worsening was not severe enough to induce high levels of stress, and participants may have been able to obtain financial support (e.g. from social welfare or from family members). It may also be possible that while we measured financial situation at midlife, it may have improved during the follow-up period.

This study has several strengths, including a large representative sample, long follow-up duration (three decades), detailed examinations for dementia diagnoses, and the possibility to control for important 
confounding factors including education and income. However, as the assessment of financial situation was self-reported, individuals experiencing negative moods might be biased in their reporting of financial situation. In this study, we had the possibility to adjust for feelings of hopelessness. As all studies, this study also has a few limitations. We did not have the possibility to adjust for financial social support, which may impact the perceptions of financial situation.

In our study, we address the long-term associations, and it might be that most of the effects from midlife to late life are mediated through perceived financial situation in late life, which was not possible to analyze. In addition, individuals' perceived financial situation might have changed during the follow-up, which may have led to an under- or overestimation of the observed effects. There may have been a survival bias; those who did not survive may have been at greater risk of dementia but we do not know their perceptions regarding their financial situation. Lastly, our measures are centered at the individual level, although many individuals rely on/share their finances with their partner or family members.

In summary, this study shows for the first time that a perceived improvement in financial situation is protective against dementia risk. It will be interesting for future research to address the underlying biological and social mechanisms for the protective role of improved financial situation.

\section{Conflict of interest}

None.

\section{Funding}

This work was supported by the following funding sources: S. S. received postdoctoral funding from the Fonds de la recherche en santé du Québec (FRSQ) under grant (27139), including its renewal (31819). She also receives funds from Alzheimerfonden, Demensförbundet, and Karolinska Institute Foundation and Funds. M. K. receives research support from The Stockholms Sjukhem foundation, Swedish Research Council for EU Joint Program Neurodegenerative Disease Research (JPND) (MIND-AD project), the Center for Innovative Medicine (CIMED), Academy of Finland, Alzheimer's Research and Prevention Foundation, Alzheimer's Association, Alzheimerfonden, AXA Research Fund, Wallenberg Clinical Scholar, Konung Gustaf V:s och Drottning Victorias Frimurarstiftelse, and ALF grant. H. Soininen receives funding from the Academy of Finland for
EU Joint Program - Neurodegenerative Disease Research (JPND) (MIND-AD project), UEF Strategic funding for UEFBRAIN, and EVO/VTR funding from Kuopio University Hospital. T. Ngandu receives research funding from the Finnish Medical Foundation.

\section{Description of authors' roles}

S. Sindi was in charge of study conceptualization, drafting of manuscript, carrying out statistical analyses, interpretation of results. I. Kåreholt contributed to study conceptualization, provided guidance on statistical analyses, and revised the manuscript and offered constructive feedback. A. DarinMattsson contributed to study conceptualization, supported with refining the methodology, revised the manuscript, and offered constructive feedback. J. Kulmala supported with refining the methodology, advised on the interpretation of results, revised the manuscript, and offered constructive feedback. $\mathrm{T}$. Laatikainen provided advice on the methodology, supported with the interpretation of results, revised the manuscript, and offered constructive feedback. $\mathrm{H}$. Soininen provided guidance on the study design, supported with the interpretation of results, revised the manuscript, and offered constructive feedback. T. Ngandu provided information on the methods and the study variables, provided advice on the methodology, supported with the interpretation of results, revised the manuscript, and offered constructive feedback. M. Kivipelto was in charge of overall study oversight, contributed to study conceptualization, supported with the interpretation of results, revised the manuscript, and offered constructive feedback.

\section{Acknowledgments}

The authors would like to thank the CAIDE participants for their time and their valuable contributions to this study.

\section{References}

Allison, P. D. (1982). Discrete-time methods for the analysis of event histories. Sociological Methodology, 13, 61-98.

American Psychiatric Association (1994). Diagnostic and Statistical Manual of Mental Disorders: DSM-IV. Washington, DC: American Psychiatric Association.

Anttila, T. et al. (2002). Midlife income, occupation, APOE status, and dementia: a population-based study. Neurology, 59, 887-893.

Bootsma-van der Wiel, A., de Craen, A. J., Van Exel, E., Macfarlane, P. W., Gussekloo, J. and Westendorp, R. G. (2005). Association between chronic diseases and 
disability in elderly subjects with low and high income: the Leiden 85-plus Study. European Fournal of Public Health, 15, 494-497.

Borodulin, K. et al. (2018). Cohort profile: the National FINRISK study. International fournal of Epidemiology, 47, 696-696i.

Butterworth, P., Cherbuin, N., Sachdev, P. and Anstey, K. J. (2012). The association between financial hardship and amygdala and hippocampal volumes: results from the PATH through life project. Social Cognitive and Affective Neuroscience, 7, 548-556.

Ebner, K. and Singewald, N. (2017). Individual differences in stress susceptibility and stress inhibitory mechanisms. Current Opinion in Behavioral Sciences, 14, 54-64.

Evans, D. A. et al. (1997). Education and other measures of socioeconomic status and risk of incident Alzheimer disease in a defined population of older persons. Archives of neurology, 54, 1399-1405.

Fischer, C. et al. (2009). Impact of socioeconomic status on the prevalence of dementia in an inner city memory disorders clinic. International Psychogeriatrics, 21, 1096-1104.

Folkman, S. (2013). Stress: Appraisal and Coping. New York: Springer.

Folstein, M. F., Folstein, S. E. and McHugh, P. R. (1975). "Mini-mental state". A practical method for grading the cognitive state of patients for the clinician. Fournal of Psychiatric Research, 12, 189-198.

Hakansson, K., Soininen, H., Winblad, B. and Kivipelto, M. (2015). Feelings of hopelessness in midlife and cognitive health in later life: a prospective populationbased cohort study. PLoS One, 10, e0140261.

Johansson, L. et al. (2010). Midlife psychological stress and risk of dementia: a 35-year longitudinal population study. Brain, 133, 2217-2224.

Johansson, L. et al. (2013). Common psychosocial stressors in middle-aged women related to longstanding distress and increased risk of Alzheimer's disease: a 38-year longitudinal population study. BMF Open, 3, e003142.

Juster, R. P. et al. (2016). Social inequalities and the road to allostatic load: From vulnerability to resilience. In D. Cicchetti (Ed.) Developmental Psychopathology (pp 381-434). New York, NY: Wiley.

Kahn, J. R. and Pearlin, L. I. (2006). Financial strain over the life course and health among older adults. Fournal of Health and Social Behavior, 47, 17-31.

Matthews, R. J., Smith, L. K., Hancock, R. M., Jagger, C. and Spiers, N. A. (2005). Socioeconomic factors associated with the onset of disability in older age: a longitudinal study of people aged 75 years and over. Social Science $\mathcal{E}$ Medicine, 61, 1567-1575.

McKhann, G., Drachman, D., Folstein, M., Katzman, R., Price, D. and Stadlan, E. M. (1984). Clinical diagnosis of Alzheimer's disease: report of the NINCDS-ADRDA work group under the auspices of department of health and human services task force on Alzheimer's Disease. Neurology, 34, 939-944.

Mirowsky, J. and Ross, C. E. (2003). Social Causes of Psychological Distress. New York: Transaction Publishers.

Morris, J. C. et al. (1989). The consortium to establish a registry for Alzheimer's disease (CERAD). Part I. Clinical and neuropsychological assessment of Alzheimer's disease. Neurology, 39, 1159-1165.
Pajak, A., Kuulasmaa, K., Tuomilehto, J. and Ruokokoski, E. (1988). Geographical variation in the major risk factors of coronary heart disease in men and women aged 35-64 years. The WHO MONICA Project. World Health Statistics Quarterly, 41, 115-140.

Palta, P., Szanton, S. L., Semba, R. D., Thorpe, R. J., Varadhan, R. and Fried, L. P. (2015). Financial strain is associated with increased oxidative stress levels: the women's health and aging studies. Geriatric Nursing, 36, S33-S37.

Pampel, F. C., Krueger, P. M. and Denney, J. T. (2010). Socioeconomic disparities in health behaviors. Annual Review of Sociology, 36, 349-370.

Pearlin, L. I., Aneshensel, C. S. and LeBlanc, A. J. (1997). The forms and mechanisms of stress proliferation: the case of AIDS caregivers. Fournal of Health and Social Behavior, 38, 223-236.

Puska, P. (2010). From Framingham to North Karelia: from descriptive epidemiology to public health action. Progress in Cardiovascular Diseases, 53, 15-20.

Sattler, C., Toro, P., Schonknecht, P. and Schroder, J. (2012). Cognitive activity, education and socioeconomic status as preventive factors for mild cognitive impairment and Alzheimer's disease. Psychiatry Research, 196, 90-95.

Shaw, B. A., McGeever, K., Vasquez, E., Agahi, N. and Fors, S. (2014). Socioeconomic inequalities in health after age 50: are health risk behaviors to blame? Social Science $\mathcal{F}$ Medicine, 101, 52-60.

Sindi, S. et al. (2016). Midlife work-related stress increases dementia risk in later life: the CAIDE 30-year study. fournals of Gerontology Series B: Psychological Sciences and Social Sciences, 72, 1044-1053.

Sindi, S., Mangialasche, F. and Kivipelto, M. (2015). Advances in the prevention of Alzheimer's Disease. F1000Prime Rep, 7, 50.

Singer, J. D. and Willett, J. B. (1993). It's about time: using discrete-time survival analysis to study duration and the timing of events. Fournal of Educational Statistics, 18, 155-195.

Southwick, S. M., Sippel, L., Krystal, J., Charney, D., Mayes, L. and Pietrzak, R. (2016). Why are some individuals more resilient than others: the role of social support. World Psychiatry, 15, 77-79.

Steffen, P. R., Walker, J., Meredith, R. and Anderson, C. (2016). The effects of job instability and financial strain on c-reactive protein in a sample of Mexican immigrants. Ethnicity \& Disease, 26, 37-44.

Steptoe, A., Brydon, L. and Kunz-Ebrecht, S. (2005). Changes in financial strain over three years, ambulatory blood pressure, and cortisol responses to awakening. Psychosomatic Medicine, 67, 281-287.

Strand, B. H., Skirbekk, V., Rosness, T. A., Engedal, K. and Bjertness, E. (2015). Income in midlife and dementia related mortality over three decades: a Norwegian prospective study. eNeurologicalSci, 1, 24-29.

Sun, F., Hilgeman, M. M., Durkin, D. W., Allen, R. S. and Burgio, L. D. (2009). Perceived income inadequacy as a predictor of psychological distress in Alzheimer's caregivers. Psychology and Aging, 24, 177-183.

Tsukamoto, K. et al. (1993). Determination by PCR-RFLP of apo E genotype in a Japanese population. Fournal of Laboratory and Clinical Medicine, 121, 598-602. 